\title{
DE ESPACIOS Y PARTICIPACIÓN: CONSTRUYENDO CIUDADANÍA EN LAS ESCUELAS MEDIAS
}

\author{
Luciana Ayelen Machado (Nacional del Comahue** \\ machadoluciana@hotmail.com
}

Recibido: 06/07/2011 Aceptado: 26/10/2011

\section{Resumen}

En este trabajo nos proponemos indagar los espacios de participación estudiantil en cuatro escuelas medias neuquinas, desde la perspectiva de la formación ciudadana. Abordamos la participación en el entorno escolar, considerando aquellas acciones, modos y aprendizajes que le permiten al estudiantado organizarse de manera colectiva para generar instancias de intervención y toma de decisiones.

Entendemos que la categoría de 'espacios de participación' nos permite considerar dicha participación como un ejercicio que combina las relaciones pedagógicas (estudiantes, docentes, organizaciones), las culturas escolares y políticas y las dimensiones física y simbólica. Estos espacios se instituyen en particulares prácticas de formación de ciudadanía en tanto que implican un compromiso con el/la otro/a, el logro de metas en común y consensos entre los sujetos que los construyen, por lo cual estas prácticas son productoras no sólo de conocimientos sino también de subjetividades.

Las siguientes son algunas de las preguntas que orientan nuestro trabajo: ¿Qué espacios de participación estudiantil se construyen, generan, propician, se apropian, en las distintas escuelas medias? ¿Qué características presentan? ¿Cómo se desarrollan los juegos de construcción-apropiación de los espacios? ¿Cuál es la potencia de dichos espacios para los/as jóvenes de hoy, para la ciudadanía actual?

\section{Palabras clave}

Escuela Media - Ciudadanía - Espacios de Participación - Estudiantes - Fuentes de Organización.

* Especialista en Currículum y Prácticas Escolares. Profesora en Ciencias de la Educación. Universidad Nacional del Comahue. Doctoranda en Ciencias Sociales. FLACSO. Becaria de CONICET. 


\section{Abstract}

In the present study we search for spaces of students' participation in secondary level schools in Neuquén Province from the perspective of citizenship education.We approach the notion of participation within school considering actions, modes and contents of learning that enable students to organize in a collective way to generate instances of intervention and decision making.

We understand that the category 'spaces of participation' allows us to consider participation as an exercise that combines pedagogical relations -students, teachers, knowledge-, schooling culture and political culture, physical and symbolic dimensions. Those spaces are instituted in particular practices of citizenship education since imply a commitment with others, the search for common purposes and consensus among the subjects involved. In this way, these practices produce not only knowledge but also particular subjectivities.

What spaces of participation are built, propitiated in the different secondary schools of our study? What characteristics do they present? How do the forms of constructionappropriation of spaces develop? What is the potency of those spaces for students today in terms of current citizenship? These are some questions that guide our work.

\section{Key words}

Secondary school - citizenship - spaces of participation - students - organization sources.

\section{Introducción}

La generación a la que pertenezco es 'hija de la democracia'. Somos hijas e hijos de la democracia (Mayer, 2009). Probablemente por ello, hemos naturalizado prácticas que, en los momentos de nuestra historia nefasta fueron castigadas y silenciadas.

También probablemente a partir de este lazo filiatorio es que me pregunto ahora -como investigadora- cómo se construyen las ideas, las acciones y los procesos que nos llevan a algunos y a algunas a comprometernos con la toma de decisiones para la vida en común y a otras/os a ser meros espectadores de ella.

Si esta familiaridad es la plataforma a partir de la cual construimos nuestro ejercicio ciudadano y, con él, nuestra participación en la esfera pública; el extrañamiento, a su vez, puede habilitarnos en el sentido de permitirnos pensar la participación, conocerla, ejercerla.

Por estos motivos, en este escrito nos proponemos indagar los espacios de participación estudiantil en las escuelas medias, desde la perspectiva de la formación ciudadana. Este análisis lo realizamos a fines del año 2009, en cuatro escuelas secundarias neuquinas atendiendo a los resultados de investigación del PAV №180 del año 2006 (1) y del trabajo de campo realizado en 2009 para 
la elaboración de la Tesis Doctoral "Los espacios de participación estudiantil en las escuelas medias neuquinas. Una mirada desde la formación ciudadana”.

\section{Escuela media y ciudadanía: dos caras de la misma moneda}

Partimos del supuesto que la institución escolar, aún en el vaivén de tensiones que la atraviesan -poniendo en duda su capacidad como 'formadora de sujetos/ciudadanía'-, constituye un espacio objeto de demandas/expectativas de distintos sujetos sociales que produce, reproduce, recrea y transforma saberes y prácticas pedagógicas constitutivas de subjetividad.

En este sentido, la escuela tiene un rol fundamental en la enseñanza de la participación entendida como la acción que nos permite involucrarnos en la vida pública y en la toma de decisiones que nos afectan como ciudadanos y ciudadanas.

Desde principios del siglo XX, la construcción del sistema educativo se ha vinculado a la formación del ciudadano; es decir, al desarrollo de capacidades que posibiliten la participación conciente en la vida política y social y el ejercicio de derechos y cumplimiento de obligaciones (Bottarini, 2007, p.170) . Éste fue el objetivo fundamental que contribuiría a la construcción del incipiente Estado- Nación, sustentada en un proyecto de principalidad del estado. Las escuelas secundarias se constituyeron, a su vez, con la función de "...disciplinar e integrar a las clases populares bajo el colectivo ciudadanos" (Pineau, 1997, p.18 citado en Bottarini, 2007, p.183); formar a las elites de la clase dirigente, para que pudieran realizar estudios superiores o desempeñar cargos en la administración pública o privada.

Hoy, al igual que en el período anterior, la educación secundaria tiene como objetivo la formación de los y las estudiantes en la construcción de ciudadanía, tal como se expresa en la Ley de Educación Nacional: “...habilitar a los/las adolescentes y jóvenes para el ejercicio pleno de la ciudadanía, para el trabajo y para la continuación de los estudios" (Ley de Educación Nacional № 26.206, 2006, cap. IV, art. 30). Sin embargo, cabe preguntarnos cómo se traduce la concreción de este objetivo en nuestras escuelas, y si efectivamente lo hace; qué acciones implica esa habilitación; qué herramientas tanto teóricas como prácticas pone a disposición para la concreción del ejercicio propuesto y las formas de transmisión.

La escuela actualmente es el único ámbito que permite el contacto sistemático con otras/os en un espacio público. Es el sitio en el cual la posibilidad de construcción de experiencias acerca de la participación invita a la juventud a vivir y construir el conocimiento y el ejercicio de la acción ciudadana; la formación ciudadana. Nos referimos a formación o educación ciudadana diferenciándola de la educación o formación para la ciudadanía ya que consideramos a las y los jóvenes como sujetos de derecho y no como quienes lo serán en un futuro. Si bien sabemos que el estatus formal de la ciudadanía se alcanza con el 
ejercicio del voto, creemos que no debe limitar el concepto el hecho de tener la edad para dicho ejercicio. Asimismo, la entendemos no sólo en los términos del conocimiento y reconocimiento de los derechos, sino, fundamentalmente, el aprendizaje del ejercicio de los mismos.

\section{Espacios de participación: sentidos y significados}

“...el colegio te da teoría, en algunos casos práctica, pero si todo eso no lo llevás a cabo no lo aprendés, no te queda en la cabeza."

(Alumna 1, Escuela $P$ )

Las palabras nos cuentan, dice Galeano, configuran nuestros mundos; nos constituyen en el decir, nos indican senderos y relatan lo que somos. Construimos palabras para leer la realidad. Transmitimos; habilitamos a quien recibe a construir sus propios sentidos, a reproducir el legado o resignificarlo. Las palabras cuentan y permiten construir significados que las expliquen, que las definan, que las identifiquen a ellas y al objeto del relato.

Pensando en nuestra necesidad de 'contar' acerca de la participación de los/as estudiantes de las escuelas medias es que decidimos que las palabras hablen acerca de ello. Espacios de participación estudiantil son las palabras que elegimos para que 'digan' sobre la participación en las escuelas. Por ello, en este artículo resignificamos aportes teóricos que colaboran en la construcción de dicha categoría.

Consideramos que el concepto de participación es complejo en tanto se construye en interacción con diversos sujetos, en sitios y coyunturas específicas. Por este motivo, la categoría de 'espacios de participación estudiantil' nos permite pensar la participación como un ejercicio que combina las relaciones pedagógicas (estudiantes, docentes, saberes), las culturas escolares y dos dimensiones: física y simbólica. De este modo, abordamos la participación en el entorno escolar, considerando aquellas acciones, modos y aprendizajes que le permiten al estudiantado organizarse de manera colectiva para generar instancias de intervención y toma de decisiones.

En otras palabras, los 'espacios de participación estudiantil' hacen referencia a redes de relaciones entre sujetos que forman parte de la institución educativa, en las que se conjugan de diversos modos los discursos, las prácticas, las normas, los rituales y los valores que generan/obstaculizan/habilitan la participación estudiantil. De esta manera, dichos espacios no sólo se construyen en relación a los lugares físicos, sino fundamentalmente en la dimensión simbólica y en la acción. Ambas dimensiones operan en la generación/habilitación de las relaciones/acciones de participación. 
'Lo físico', está vinculado a la topografía del lugar que se pretende describir; es decir, mapa topográfico y temporal de los ámbitos concretos, visibles, dimensionables y limitados. Esta dimensión, pensada en clave escolar, nos invita a reflexionar acerca de la ocupación y circulación en el aula; la disponibilidad de la institución para la realización de actividades extra-escolares, por parte del estudiantado; la ocupación y circulación por los lugares de la escuela y los cronogramas escolares.

'Lo simbólico', por su parte, implica el entramado de significaciones constituidas por normas, valores y modos de relaciones. Estas relaciones se convierten en redes en las cuales se entrelazan los discursos y en ellos el poder, convirtiéndose así en construcciones dinámicas, en permanente transformación. La dimensión simbólica se constituye a partir de las relaciones que se construyen en torno a los contenidos curriculares en relación a la participación; las organizaciones y los reclamos estudiantiles; las actividades áulicas; los actos escolares; los discursos de docentes y de alumnas/os.

Las relaciones que conforman los espacios de participación estudiantil se producen ante la ocasión de tomar parte de algo, provocando acciones que llevan a la intervención en asuntos públicos, como instancias de construcción de lo común para la toma de decisiones. Estos espacios se instituyen en particulares prácticas de formación de ciudadanía en tanto que implican un compromiso con la/el otra/o, el logro de metas en común y consensos entre los sujetos que los construyen, por lo cual estas prácticas son productoras no sólo de conocimientos sino también de subjetividades.

Pensar en la participación, nos remite a las acciones de la ciudadanía en la esfera de 'lo público'. Con este término nos estamos refiriendo a un espacio complejo, donde se construye lo común, en el que las particularidades tienen lugar, pero que deben articularse en construcciones colectivas que las incluye y las excede (Dussel y Southwell, 2006, p.29).

La participación no sólo es un derecho constitucional, sino también un medio para mejorar la convivencia. Participar implica no sólo ser parte, en referencia a la identidad, a la pertenencia de los sujetos, sino también tener parte, a partir de la conciencia de los deberes y derechos, comprometerse cotidiana y permanentemente y tomar parte, en relación al logro de acciones concretas (Oraisón y Pérez, 2006); implica un compromiso con el/la otro/a, concierne metas en común, consenso y toma de decisiones para alcanzar dichas metas.

Si bien el sentido común parece reducir la noción de participación estudiantil a los centros de estudiantes y a las diversas organizaciones estudiantiles, en nuestro trabajo la visión se complejiza y amplía. Pensamos en espacios que dan lugar a múltiples interacciones y dinámicas entre alumnado y profesorado, que permiten el desarrollo de competencias para el ejercicio de la vida ciudadana. 
De este modo, definimos los espacios de participación como una categoría relacional que alude a una dimensión simbólica, un nudo de sentidos y un lugar en el cual se despliega -dimensión física-. Estos espacios se constituyen en interacciones, lo cual les otorga un carácter dinámico y procesal. Los espacios se construyen, se reproducen, se proponen, circulan, se apropian, se expropian, se otorgan, se ceden, se negocian, se aceptan.

Teniendo en cuenta que los espacios de participación, tienen como escenario principal las escuelas y como sujetos al estudiantado debemos pensarlos como una particular práctica pedagógica-política. Es decir, si la educación es un acto político en términos que constituye una práctica social de producción, reproducción y transformación de conocimientos y sujetos es posible pensar los espacios desde una perspectiva pedagógica como particulares prácticas de formación ciudadana, en tanto productoras de conocimientos y subjetividades. Las formas en que los conocimientos son producidos y comunicados y cómo los/ as estudiantes participan en el proceso son aspectos políticos fundamentales para tener en cuenta (Hernández, 1997).

Llevando estas definiciones al ámbito escolar y considerando la escuela como el primer ámbito de actuación pública del alumnado, como aquella que ofrece oportunidades de relacionarse con la normatividad institucionalizada en la que se especifican deberes y derechos (Rodríguez, 1997), es que podemos pensarla como un espacio de construcción de experiencias para la vida en democracia (Frigerio y Diker, 2005), como espacio público de construcción de lo público (Cullen, 1997).

A la vez que procuramos su definición, entendemos que los distintos modos de configuración de los espacios de participación estudiantil conforman y son conformados por una particular cultura escolar.

Cada una de las escuelas produce sus propias prácticas y sus discursos; por lo tanto, las culturas escolares configuran distintos espacios de participación al interior de las instituciones educativas, a la vez que éstos contribuyen a la producción de dicha cultura. Para definir este concepto tomamos las palabras de Viñao Frago (2002) quien entiende la cultura escolar como “... un conjunto de teorías, ideas, principios, normas rituales, hábitos y prácticas (...) sedimentadas a lo largo del tiempo en forma de tradiciones, regularidades y reglas de juego no puestas en entredicho y compartidas por sus actores, en el seno de las instituciones educativas..." (p. 38). Estas regularidades perduran en el tiempo, permitiendo la construcción de estrategias que habilitan la acción en la cotidianeidad escolar. La perdurabilidad de la cultura escolar permite la construcción de estrategias que posibilitan la acción en la cotidianeidad escolar.

A partir de lo antedicho, estamos en condiciones de afirmar que los espacios de participación estudiantil se convierten en parte constitutiva de la cultura escolar, así como también son constituidos por ella. Las redes de relaciones de los actores institucionales que configuran los diversos espacios, son constituidas 
por -y constituyentes de- los discursos, las prácticas, las normas, los rituales y los valores de las distintas escuelas medias de la provincia de Neuquén. Por ello, pensar en los espacios de participación de los y las estudiantes de las escuelas medias, en vínculo con la cultura escolar, nos orienta a resignificar sus elementos estructurantes, generadores, habilitantes u obturadores de dichos espacios.

\section{Espacios de participación estudiantil: delineando límites}

“... el centro de estudiantes, el cuerpo de delegados, capaz que tendríamos que aprender a usarlos mejor (...), porque por ejemplo el centro de estudiantes lo puede aprovechar cualquier persona y a los delegados también, capaz que no los sabemos utilizar, como que quedamos ahí nomás"

(Alumna 1- Escuela P)

A partir del análisis del trabajo de campo realizado en el año 2006, delineamos los límites que habilitan la visibilización de distintos espacios: Según fuentes de organización, gestionados por alumnos/as u organizados por los/ as docentes; según la temporalidad, esporádicos o duraderos; según la mirada pedagógica, prescriptiva o contingente y según los bordes escolares, el adentro y el afuera escolar.

En este escrito, sólo abordamos uno de los criterios de clasificación de los espacios de participación estudiantil: el que refiere a las fuentes de organización. Aquellos que atienden a la pregunta por quiénes generan, crean, propician, la construcción de dichos espacios, procurando diferenciar entre los espacios que son generados/organizados por la institución educativa, de aquellos que lo son por el estudiantado como así también las tensiones posibles entre ellos. (2)

Tradicionalmente, en las escuelas los equipos directivos han ordenado, generado y promovido las distintas actividades del estudiantado. Actualmente, como veremos en los siguientes apartados, en algunas de las instituciones analizadas, el estudiantado es parte activa en la construcción de la cultura escolar, en permanente negociación con la gestión institucional.

\section{Fuentes de organización: estudiantes}

Según lo dichos de las/os entrevistadas/os, podemos identificar al menos dos grandes tipos de sujetos alumnos/as que construyen, en interacción, los espacios de participación. Por un lado, aquellos/as que no intervienen en ninguna organización extra escolar; por otro, quienes que tienen participación en organizaciones por fuera de la escuela como partidos o movimientos políticos o algún/a integrante de su familia lo hace. 
Los espacios generados/gestionados por alumnos y alumnas que no participan en acciones extra-escolares, generalmente se desarrollan en el ámbito áulico, relacionados con temáticas puntuales y eventuales.

En las cuatro escuelas del estudio hay alumnas/os que sobresalen por su carisma, la empatía con sus pares y/o capacidad de expresión verbal fluida. Ellos y ellas son quienes organizan y llevan adelante los pequeños y esporádicos espacios de participación en los que las interacciones entre el alumnado se desarrollan en pos de la concreción de objetivos comunes. Algunos de estos espacios son los que, al momento de las entrevistas, se habían conformado en las escuelas para la compra del buzo identificatorio del curso de $5^{\circ}$ año y la organización de la fiesta de egresados/as.

Por ejemplo, en la escuela C (3), un grupo de alumnas gestionó la fiesta de egresados/as, una de ellas cuenta que quienes organizan los espacios son "...los que más hablamos o los que más estamos relacionados con todos, los más tímidos esos nos siguen." (Alumna 1- Escuela C).

Como éstos, hay otros espacios que habilitan la voz de las y los estudiantes en la discusión con otra y con otro que piensa igual o diferente a ellas/os, permitiendo la búsqueda de pequeños consensos. Son espacios generados donde tradicionalmente no existían o les eran negados a las/os alumnas/os. Son espacios que amplían el margen para la acción del estudiantado y permiten un mayor involucramiento en la gestión institucional (Cornwall, 2002).

Algunos de ellos son los que en la escuela P (4) llaman 'intervenciones'. Éstas son acciones teatrales con las que un grupo de alumnos/as del centro de estudiantes en conjunto con otro de primer año conmemora alguna fecha:

Hemos hecho intervenciones, por ejemplo para la noche de los lápices poníamos unos cartelitos en los bancos que decían Francisco López se sentaba acá (...) por ahí entrábamos nosotros como si fuera una movilización pidiendo el boleto estudiantil por la noche de los lápices pero bueno siempre tenemos la misma respuesta 'eh que embole', pero mientras haya uno o dos... (Alumno 2- Escuela P).

Consideramos a estas las intervenciones como espacios de participación por ser el producto de interacciones entre algunos y algunas de los/as estudiantes, buscando modificar algo en la/el otra/o -par- , en este caso convocando a la reflexión a partir de un hecho puntual sobre un determinado suceso.

Además, en esta escuela se generan debates áulicos en las horas libres o en horas de clase ante algún tema de interés. También generan asambleas, proponiendo a los/as coordinadores/as, docentes y/o equipo directivo un encuentro con la otra parte involucrada para dirimir algún asunto específico, sin la mediación de adultos.

En contrapartida con los espacios que se construyen en esta institución, en líneas generales, en las escuelas C y D (5) observamos dificultades en la 
objetivación de los modos de organización de actividades. Podríamos aventurarnos a pensar que a los y las estudiantes de estas instituciones les resulta difícil verse a sí mismos/as como sujetos de la organización, como gestores de sus propios espacios, de la acción. Identifican fácilmente actividades instituidas, como la compra de los buzos o la organización de la fiesta de egresados/as, pero no señalan como actividades organizadas por ellas/os aquellas que son necesarias crear para dar respuesta o solución a alguna problemática puntual como reclamos por calefacción.

Por otro lado, en todas las escuelas hay estudiantes que tienen algún tipo de familiaridad con la participación, ya sea por formar parte de alguna agrupación fuera de la escuela o por ser hijos o hijas en familias activas en intervenciones en los espacios públicos. En ocasiones, ambas características coinciden.

En relación a las alumnas y los laumnos que promueven los espacios con algún tipo de trayectoria en actividades de participación extra escolar, podemos afirmar que son quienes generalmente lideran los Centros de Estudiantes. En la escuela $\mathrm{D}$, por ejemplo, el padre del presidente del Centro de Estudiantes es militante del gremio docente de la provincia.

Dos de los alumnos entrevistados en la escuela $\mathrm{P}$ son integrantes del centro de estudiantes. La madre de uno de ellos ha militado en diversas fuerzas políticas. Fuera de la escuela, este alumno participa en la Coordinadora de Estudiantes Secundarios, en representación del Centro de Estudiantes de su escuela. El otro, colabora en un merendero de un barrio periférico de la ciudad.

En estos estudiantes aparecen recursos simbólicos y sentimientos de empoderamiento y compromiso con la participación que pueden ser leídos como huellas de su particular entorno familiar en interacción con la política local.

Un espacio de participación estudiantil de relevancia en las escuelas son los centros de estudiantes. Es el espacio de privilegio reconocido por las/os entrevistadas/os como generador de actividades cuando se les pregunta por las oportunidades de participación que tienen. En las instituciones que existen centros de estudiantes -J (6), P y D-, fue un grupo de alumnas/os quienes gestionaron las elecciones. En este punto creemos que es interesante interrogarnos acerca de la importancia de la representación estudiantil, en términos de quién habla y por quién se habla en los espacios de participación.

Las elecciones de la escuela $\mathrm{P}$, al igual que en $\mathrm{J}$, fueron con la participación general del estudiantado por medio de votaciones obligatorias, en el horario de clase. Las alumnas y los alumnos comentan que previamente al día del sufragio se realizaron asambleas y se repartieron panfletos con las propuestas de cada una de las listas. Como podemos ver, en estas escuelas son las/os mismas/ os estudiantes quienes propician formas democráticas de participación, son quienes construyen espacios democráticos de participación estudiantil, donde la posibilidad de voto, de elección es el resultado de un proceso de escucha, 
reflexión, toma de decisiones y aceptación de los resultados, tanto de manera individual como colectiva. Además, esta experiencia de participación les brinda a los/as estudiantes una herramienta más para el ejercicio de la ciudadanía.

En la escuela J, en las votaciones del centro de estudiantes vigente al momento de las entrevistas se había presentado sólo una lista y los/as alumnos/as tenían la posibilidad de votar en blanco en caso de no querer su constitución. En la escuela $\mathrm{D}$ ocurrió un hecho similar, aunque difirió la forma en que se tramitó la elección: "En realidad se eligió medio así como que dijeron nosotros somos el centro porque no había nadie que se haga cargo, no había ninguna lista, así que se encargaron los chicos, son del curso" (Alumna 1- Escuela D).

Estas palabras son sumamente interesantes para pensar las relaciones al interior de los espacios de participación en esta escuela. A partir de los dichos de las y los estudiantes, ¿podemos decir que éste es un espacio de participación? Si consideramos que los espacios de participación estudiantil son construcciones en las que el estudiantado, en interacción, toma decisiones que le afectan en la vida común, no podemos considerar un espacio de participación a la autodesignación de un grupo de estudiantes para representar a sus compañeros/ as; aquí cabe la pregunta por la legitimidad de dicha representación.

El análisis nos indica que cada institución genera sus propias reglas y, con ellas, sus propios espacios de participación. La regla general que apela a la tradición señala que el centro de estudiantes debe ser elegido por medio del voto del estudiantado; sin embargo, como podemos observar, en esta escuela las situaciones ocurren de modo distinto a lo esperable, alguien -un grupo- se instala y perdura en el tiempo, convirtiéndose en el representante legítimo de sus pares ya que en las entrevistas es reconocido como tal. De este modo, estaríamos ante una legitimidad obtenida y otorgada por acuerdos situacionales y no por reglas trascendentales.

Si bien en la escuela J estamos ante una elección de Centro de Estudiantes democrática, no son menores las observaciones que hacen dos de los entrevistados en relación al desempeño y la representación de sus integrantes:

En el centro de estudiantes se encuentran amigos (...) todos tienen la misma opinión y te dicen somos mas y pensamos así (...) eso me pasaba a mi (...) yo tengo un pensamiento formado y vos querés opinar un poquito y te dicen somos más, es la democracia media mala porque no toman en cuenta tu opinión, te escuchan pero siguen haciendo lo que estaban haciendo. (Alumno 1- Escuela J).

Sin lugar a dudas, la voz de estos alumnos nos alertan acerca de la representación de las minorías. El alumno denuncia la falta de consideración de su palabra, de su opinión, lo cual nos lleva a cuestionarnos acerca de la democratización de los espacios de participación, de la circulación y autorización de la palabra dentro de ellos y del monopolio de la toma de decisiones. 
En este sentido, nos parece interesante hacernos la pregunta por la posesión de la información ya que los sujetos que concentran la información son quienes suelen tener la posibilidad de tomar las decisiones que conciernen al grupo estudiantil. Incluso, en algunos casos se observa la distribución de la información y la recepción de sugerencias -como en las asambleas que se desarrollan en la escuela J que veremos en siguientes apartados- pero la toma de decisiones se concentra en unas/os pocas/os.

Estas relaciones de poder que dejan al descubierto el predominio de unos sujetos por sobre otros/as, lejos de estimular la participación producen un rechazo y posterior abandono de los espacios (Kriger, 2010). ¿Quiénes representan a quiénes?

Alguna de las actividades emprendidas por el centro de estudiantes que pueden constituirse como espacios de participación, es la que comentan estudiantes de la escuela $P$ en relación a la organización de una encuesta que se aplicó para todo el alumnado a través de la que se buscaba decidir si poner el nombre de Carlos Fuentealba (docente asesinado en un corte de ruta por reclamo salarial, en el año 2007) a alguno de los ámbitos de la institución. Esta actividad se consolidó como un espacio de participación estudiantil porque implicó a la totalidad de estudiantado en una toma de decisiones que afectaba no sólo a la escuela sino también a la comunidad.

Otro espacio cuya única fuente de organización son las alumnas y los alumnos, es el cuerpo de delegados. Las delegadas y los delegados son parte del Centro de Estudiantes -excepto en la institución $C$ en la que no está conformada dicha organización- por lo cual estaría garantizada la representación de todas/os las/os alumnas/os de las escuelas. De este modo, la legitimidad de las representaciones y autorizaciones se construye al interior del mismo claustro. En las cuatro escuelas el cuerpo de delegados/as se elige por votación; sin embargo, al momento de las entrevistas, en las dos escuelas públicas sólo se postuló un compañero en $\mathrm{J}$ y una compañera en $\mathrm{C}$, por lo cual no hubo elecciones.

En la escuela C, una alumna afirma que las/os delegadas/os se eligen por afinidad y que no se tiene en cuenta la responsabilidad de quienes son elegidas/os.

Está bien lo que hacen por votación, creo que tendrían que ser mas concientes a quién eligen y a quién no, a los chicos más estudiosos nunca los eligen porque no me hablo con él, porque no me llevo con él, pero sabés que es responsable, y no elegir al chanta, al mas alegre del curso. (Alumna 1- Escuela C).

Si la función del/a delegado/a es tanto la de representar a sus pares/as como la de transmitir información de distintas fuentes, ¿qué condiciones parecen reunir? En la expresión de la alumna encontramos rastros de la identificación 
adolescente, de la empatía entre pares, de habilidades comunicativas. Desde el sentido común, la alumna apela a la toma de conciencia sobre la necesidad de elegir representantes responsables, pero finalmente se eligen por condiciones comunicativas o histriónicas.

En la escuela privada laica podemos identificar algunos indicios de una representación diferente a la que analizamos previamente. Teniendo en cuenta quiénes poseen la información necesaria para tomar decisiones por lo cual el conocimiento se convierte en una herramienta fundamental no sólo de la representación, sino principalmente del ejercicio de poder, nos parecen relevantes los señalamientos realizados por los/as estudiantes. Coinciden en afirmar que los/as representantes elegidos/as son los/as estudiantes más comunicativos y más críticos "...porque tiene que poder comunicar lo que piensa el curso..." (Alumna 1- Escuela P). Acá podríamos pensar que la acción de elegir un/a representante implica una concientización mayor que en la escuela $\mathrm{C}$, en relación a la importancia de su función.

La posibilidad de levantar la voz parece ser otra característica de los/as representantes en el cuerpo de delegados/as que se suma a la responsabilidad, a la empatía y la habilidad comunicativa. "Siempre buscamos elegir a los que sabemos que van a hacerse escuchar..." dice la alumna 3 de la escuela J. Como podemos notar, estas particularidades diferencian a las escuelas entre sí lo que, sumado a los rasgos de otros espacios de participación en cada una de ellas, nos permiten la identificación de particulares culturas escolares y, con ellas, determinadas miradas hacia la formación ciudadana. Estas características dicen mucho acerca de las relaciones entre docentes y estudiantes, así como también son un reflejo de la concepción de participación que se sostiene en tanto 'condiciones necesarias para ejercer la representación'.

\section{Fuentes de organización: docentes}

En cuanto a los/as espacios generados por el profesorado, podemos diferenciar entre aquellos en los que la participación es obligatoria y los que permiten que los y las estudiantes tomen la decisión de participar o no.

Otra de las fuentes de organización de los espacios de participación estudiantil la constituye el profesorado (docentes, equipo directivo, asesoras/es pedagógicas/os). Los adultos de las escuelas promueven distintas actividades que, en ocasiones, los podemos identificar como espacios de participación estudiantil. En algunos casos, dichos espacios comienzan por gestión de docentes y continúan liderados por el alumnado; en otros, los adultos son quienes los sostienen en el tiempo. Varios, se organizan dentro del horario escolar, mientas que otros -generalmente optativos- se constituyen en actividades extra-curriculares. Algunos espacios gestionados por el profesorado invitan a la participación y la hacen efectiva, son reales; otros, son simbólicos ya que el estudiantado tiene presencia pero su voz no es parte de las tomas de decisiones que allí se promueven. 
Estos espacios de participación construidos por docentes, convocan a la participación de los alumnos y de las alumnas. Estos espacios no siempre posibilitan el empoderamiento de los y las invitados/as, lo cual depende de la gestión que convoca.

En este sentido, los discursos de los y las estudiantes de las escuelas $\mathrm{J}$ y $\mathrm{P}$ muestran que el profesorado -en la mayoría de las ocasiones el equipo directivo- propone instancias que se consolidan como espacios de participación estudiantil, tales como asambleas generales y/o por curso, participación en la elaboración las pautas áulicas y encuentros al ingreso de la jornada. Se construyen como acciones que surgen desde los/as docentes, pero en los que los y las estudiantes tienen una participación activa en la toma de decisiones.

En ambas instituciones se promueve la elaboración de pautas áulicas junto a los/as docentes de cada asignatura, permitiendo a los y las estudiantes ser artífices de las normas que los/as regulan por lo cual este espacio puede convertirse en un sitio de posibilidad, de búsqueda y expresión y de construcción de sus propias leyes.

Si arriba analizamos la legitimidad de los espacios en términos de la representación, ahora aquí podemos preguntarnos por la legalidad y la legitimidad en tanto regulaciones construidas por las/os reguladas/os. Lo legal, en cuanto a saberse sujetos/as de derecho reposicionándose en relación a sus actos y su responsabilidad. Lo legítimo al ser partícipes de la elaboración de las normas que las/os regulan estas regulaciones requieren y adquieren de una mayor responsabilidad.

Por otro lado, la dirección de la escuela J, ante la necesidad de informar algunas cuestiones particulares, propone la realización de asambleas. En ellas, los y las estudiantes pueden opinar, debatir y tomar decisiones por medio de votaciones. Sin embargo, la palabra suele ser tomada por los representantes del centro de estudiantes y, por este motivo, las/os entrevistadas/os aseguran que no suelen opinar en dichos espacios a pesar de tener la posibilidad abierta de hacerlo: "Es un espacio para todos, casi nadie lo utiliza, por ahí más que nada con el centro de estudiantes para comentar alguna cosa." (Alumna 2Escuela J).

Las asambleas de tomas de decisiones, no sé, como poner un espejo en el baño, no sé, hacen una asamblea, a ver si están todos de acuerdo, si todos quieren colaborar... para el tema de las notas se hicieron asambleas... para todo lo que haya que tomar decisiones se organizan (Alumna 3-Escuela J).

En las voces de estas estudiantes se expresan dos cuestiones. Por un lado, que no es un espacio utilizado por la totalidad del alumnado, sino principalmente por las y los integrantes del centro de estudiantes. Por otro, que la alumna 3 no lo asume como propio ya que se coloca por fuera de dicho espacio. Estas asambleas parecen ser espacios apropiados sólo por pocos/ as. Espacios invitados, concurridos pero poco aprehendidos por parte de los y las estudiantes. 
Es notorio cómo en esta escuela los y las estudiantes reconocen sus espacios de participación, así como también admiten que no les dan la utilidad que podrían. Esto puede leerse desde varios puntos de vista, es más, como ya dijimos, las/os mismas/os alumnas/os lo analizan. Algunas/os refieren a diferencias de personalidad, habilidades para la expresión/timidez; otras/os aluden a que es una decisión individual y otros/as hacen referencia al interés. No está demás aclarar que estas miradas acerca de la falta de participación son comunes a las cuatro instituciones.

Hay dos actividades en la escuela $D$ que son organizadas por la escuela y a las que los alumnos y las alumnas remiten cuando se les pregunta acerca de su participación: el grupo de Líderes y la Misión. El primero lo organiza una preceptora y está conformado voluntariamente por alumnos/as de $3^{\circ}$ a $5^{\circ}$ año. Su finalidad es la preparación de los/as líderes para la concurrencia a los campamentos de estudiantes más chicos/as, en calidad de acompañantes de los grupos.

Se llama 'Misión' a un viaje organizado por la institución, que realizan los y las estudiantes de $5^{\circ}$ año hacia algún lugar de escasos recursos. Llevan ropa, alimentos y colaboran con la comunidad a la que van. Ésta es una actividad extracurricular que puede ser analizada desde el lugar que la escuela le da al/ la otro/a como objeto de caridad. No es nuestra intención aquí cuestionar el ideario social del colegio, sino centrarnos en por qué consideramos que estas actividades podemos identificarlas como espacios de participación, invitados por la escuela.

Las/os entrevistadas/os recurren a estas dos actividades cuando se les pregunta por las oportunidades de participación que les ofrece la escuela. La concurrencia es optativa en ambas actividades extracurriculares, lo cual implica un compromiso y una dedicación más allá de las horas de clase. En algunos casos, la pertenencia a dichos grupos es una herencia familiar, como lo es la concurrencia a esta institución; probablemente ambos legados se sostengan sobre la base de la fe católica y la caridad y/o asistencia.

Otros espacios de participación gestionados por docentes, no obligatorios, son las marchas realizadas con motivo de diversos reclamos. Las mismas se encuentran estrechamente vinculadas a lo que interpretamos como motivación proveniente de sentimientos de 'defensa' de lo propio, como en el caso de reclamos por seguridad (Escuela $\mathrm{C}$ ) o de reclamos por deficiencias edilicias (Escuela J). La respuesta de ambas escuelas ante esta situación de defensa de lo propio, fue diferente. Por ello, podríamos suponer que la motivación se encuentra estrechamente ligada al sentido de pertenencia, a la identificación.

En la escuela $\mathrm{C}$ la dirección convocó a alumnos, alumnas y familias a una reunión y marcha por la inseguridad que afectaba a la comunidad escolar. A pesar de que una numerosa concurrencia tomó la decisión de las acciones a seguir, fueron sólo unas/os pocas/os junto a la directora las/os que marcharon hacia el Consejo de Educación a reclamar por mayor seguridad: 
Se decidieron entonces cortar las clases y hicimos una marcha al consejo todo, y los que fueron en total todos alumnos, padres y docentes no se si llegábamos a 15, de los 500 supuestamente que son en los dos turnos (Alumno 1- Escuela C).

En cuanto a los relatos de la escuela J nos muestran que este espacio de participación convocado por la escuela tuvo una respuesta masiva, tanto en lo que refiere a la toma de decisiones respecto de los pasos a seguir como a la acción concreta de la movilización:

Estábamos ahí toda la escuela en el SUM, y el director dijo 'chicos pasa esto (...), alguien tiene una propuesta?' (...) y se propuso: que la arreglen los alumnos, no nos dejan, que la arreglen los profesores, no se puede (...) yo me acuerdo que salieron un par de alumnos y dijeron qué tal si hacemos una nota (...) la firmamos todos. (...) sé que no la contestó nadie, se elevó otra nota, tampoco la contestaron. Se hizo la tercera, no la contestó nadie, entonces se dijo "hagamos una manifestación, así nos van a escuchar" (...) fueron todos y al final lograron que vengan a arreglarlo, y tuvimos calefacción otra vez, y decisiones así hemos tomado (Alumna 3- Escuela J).

En el caso de esta escuela coincidimos con Rossi (2009) en que "....ante coyunturas críticas (...) existe el potencial para la emergencia de conciencias grupales que sostengan la acción colectiva entre jóvenes..." (p. 133). No obstante, esta afirmación se desvanece al considerar los acontecimientos de la escuela C. En estos casos las familias son invitadas por las escuelas a participar en pos de un bien para la sus hijos/as. ¿Cómo juega aquí el sentimiento de pertenencia de las familias para con las escuelas?

Teniendo en cuenta las características de ambas escuelas y sus contextos, encontramos que las diferencias que se presentan en estas situaciones pueden ser una consecuencia de la especificidad de cada escuela. Recordemos que la escuela $J$ es una institución ubicada en una localidad del interior neuquino con fuertes vinculaciones con el ejército. En ese contexto, la escuela es cuestionada por algunas/os de las/os entrevistadas/os por lo que entienden como 'ideología de izquierda' manifiesta en las prácticas cotidianas. Sin embargo, tanto los/ as alumnos/as como sus familias la valoran por el seguimiento del alumnado, por la originalidad de la modalidad y por la enseñanza de corte humanístico que sostiene a pesar de su perfil técnico. Éstas pueden ser algunas de las razones por la cual la comunidad escolar se hace presente ante la necesidad del reclamo.

La escuela C se presenta inserta en un contexto hostil, agresivo, por el cual reclaman una solución ya que ni siquiera los muros de la escuela salvaguardan a sus estudiantes de la violencia. Probablemente esté presente en el ausentismo a la marcha -valga la contradicción- la sensación de que nadie va a escuchar el 
reclamo o, incluso, que no hay posibilidad de cambio de la situación en la que viven permanentemente en el barrio y a la que la escuela no es ajena.

Como hemos señalado, algunos de los espacios de participación estudiantil que se configuran en las escuelas, se encuentran mediados por la acción de los adultos de las instituciones educativas. Dichos espacios -como las asambleas de la escuela J- están, incluso, dirigidos/coordinados por los adultos. Asimismo, en otras o iguales instancias, la gestión se basa en una aparente participación, reduciendo la misma a un mero formalismo institucionalizado en la consulta sin concretar acciones en consecuencia con las expresiones de los y las adolescentes.

Creemos que el lugar de los adultos en la tarea de enseñanza es crear las condiciones para que los y las estudiantes sean sujetos activos en la construcción de ideales o de un proyecto. Considerando esta afirmación, es necesario que nos preguntemos si estas intervenciones 'adultas' pueden ser consideradas como generadoras de legítimos espacios de participación de los/ as estudiantes o si las fronteras de dicha categoría limitan el concepto abordado desde una mirada de la participación entendida como participación simbólica y no como real. Es decir, si son espacios reales de participación o lo son sólo en apariencia; si las voces de las/os invitadas/os son efectivamente escuchadas o tienen influencias ilusorias. Si los sujetos que conforman los espacios de participación son participantes o integrados.

En este tipo de participación en torno a las relaciones que construyen espacios donde interactúan los adultos con el estudiantado, se pueden identificar algunos en los que los/as estudiantes son sólo consultadas/os, reduciendo su status al de consumidores/as de servicios y no como participantes activas/os en la toma de decisiones; desplazándolos/as de su lugar de ciudadanas/os, de sujetos de derecho.

\section{Elucidaciones e interrogaciones}

"...depende de cada uno si participa, los espacios están dados y cada uno elige."

(Alumno 1- Escuela D)

"Todos tienen la posibilidad de aquí que quieran tomar esa posibilidad es distinto."

(Alumno 2- Escuela J)

Las frases de estudiantes que utilizamos de epígrafe para este último apartado nos remiten a la pregunta acerca de la no participación. Hemos visto en las páginas anteriores que los espacios de participación estudiantil pueden ser organizados por el alumnado y/o por el profesorado. Sin embargo, también 
pudimos ver que no todas/os tienen parte en las tomas de decisiones que cada uno de estos espacios promueve. Esto nos permitió reflexionar acerca del lugar del alumnado en la toma de decisiones, así como también del agenciamiento de los y las estudiantes, ambos necesarios para el ejercicio de una participación real en la esfera pública.

Observamos en los discursos estudiantiles que en las escuelas C y D son los adultos quienes mayormente promueven los espacios de participación estudiantil, lo cual sucede de manera diferente en las escuelas $\mathrm{P}$ y $\mathrm{J}$ donde los y las alumnos/as se saben sujetos activos y autónomos en la creación de sus propios espacios de participación. Éstos son construidos por y para estudiantes que deciden formar parte de ellos y asegurarse que perduren siempre que exista el interés de hacerlo. Podemos analizarlos, a su vez, como posibles espacios de resistencia por ser ideados y gestionados por el alumnado, logrando así una legitimación de sus acciones y la sensación de empoderamiento en la concreción de las mismas.

Unos y otros dan lugar a voces de resistencia o protegen el statu quo, dependiendo de las formas de agenciamiento. En este sentido, tanto los docentes como algunos/as estudiantes de las escuelas $\mathrm{P}$ y $\mathrm{J}$ promueven espacios de participación que podrían habilitar la formación de habilidades en su alumnado. Sin embargo, vimos en los análisis que la participación real es de unos/as pocos/ as, generalmente los integrantes de los Centro de Estudiantes.

El desconocimiento de los derechos atenta contra la posibilidad de los y las estudiantes de convertirse en agentes de participación. Asimismo, la ausencia de instancias concretas de participación, inhibe el ejercicio efectivo de los derechos, reduciéndolos a mero contenido curricular. Considerar al estudiantado sólo en su condición de futuras/os votantes y darles herramientas escasas para ejercer la ciudadanía no sólo en su dimensión formal, sino también comunitaria, dice mucho acerca de la cultura política que se promueve.

Según nuestra forma de entender los espacios de participación, su construcción y apropiación no puede desligarse del conocimiento acerca de la posibilidad de gestión y de la concepción de participación que sostienen los/as alumnos/as. Para que los espacios de participación se construyan, el estudiantado debe saberse hacedor de los mismos, con capacidad de creación, de voz y de injerencia institucional. De otro modo, estaríamos concibiendo a los y las estudiantes como meros consumidores de la educación que se les ofrece en lugar de agentes de cambio, de ciudadanas y ciudadanos activas/os.

Consideramos que las instituciones educativas son fundamentales en la formación ciudadana, en las transmisiones de formas de ciudadanía y, con ello, de herramientas para el ejercicio de la participación. En este sentido, cada uno de los espacios de participación que hemos identificado en las escuelas se constituyen en instancias de formación ciudadana en tanto implican un sentimiento de responsabilidad para con la/el otra/o, la consecución de metas comunes y de pequeños acuerdos entre los sujetos involucrados. 
Las miradas al camino recorrido, nos acercan indicios y nos abren nuevas preguntas para seguir adentrándonos en los espacios de participación estudiantil: ¿Qué discursos sobre ciudadanía se construyen? ¿Cómo se articulan, en los espacios de participación escolar, los procesos históricos, culturales, políticos y económicos de la provincia de Neuquén? Tarea para próximos escritos.

\section{Notas Bibliográficas}

(1) EI PAV No 180 "Intersecciones entre desigualdad y educación media- un análisis de las dinámicas de producción y reproducción de la desigualdad escolar y social en cuatro jurisdicciones.", fue coordinado por el Área de Educación de la FLACSO y efectuado en forma conjunta por equipos de investigación pertenecientes a: Ministerio de Educación De la Ciudad Autónoma de Buenos Aires y Universidades Nacionales de Salta, de Comahue y de La Plata. El análisis realizado en este trabajo de investigación nos brinda las primeras líneas exploratorias a partir de las que definimos el problema y los ejes de la Tesis de mi autoría en el marco del Doctorado en Ciencias Sociales de la FLACSO, de la cual se construye el presente escrito. Además este trabajo se inscribe en el Proyecto de Investigación "Escuela Media y ciudadanía. Un estudio sobre prácticas pedagógicas en relación a la vida política y a la vida productiva en la Provincia de Neuquén" de la Facultad de Ciencias de la Educación, Universidad Nacional del Comahue.

(2) Si bien la construcción de las mencionadas categorías se elaboró con el análisis realizado sobre entrevistas y encuestas del año 2006, en el año 2009 se realizaron 24 nuevas entrevistas, 6 por escuela, a partir de las cuales efectuamos el análisis de cada una de dichas categorías. Esto se debió a la necesidad de profundizar en la temática de la participación que no se abordaba directamente en el primer trabajo de investigación. Por lo tanto, las conclusiones que aquí se presentan están desarrolladas en base a las voces de 3 alumnos y 3 alumnas del último año de estudio de cada una de las cuatro escuelas seleccionadas.

(3) Esta escuela es pública, con orientación en Perito Mercantil. Se ubica en un barrio periférico de la capital neuquina, atendiendo a una población aproximada de 400 alumnos/as de bajos recursos y problemáticas sociales diversas, cuyas familias, en muchos casos, son beneficiarias de planes provinciales. El barrio es visto por docentes y directivos como un barrio 'peligroso' por el alto nivel de adicciones existentes y, como consecuencia de esto, los elevados índices de violencia.

(4) La escuela $P$ es pública de gestión privada, que atiende a 350 alumnos/as de sectores medio-altos de la ciudad de Neuquén. Tiene dos orientaciones: Ciencias Sociales y Ciencias Exactas, desarrollándose el trabajo de campo en la primera de ellas. Sus estudiantes son hijos/as de familias de profesionales y/o funcionarios/as del gobierno provincial. Cuenta con un esquema curricular que difiere de la de las otras escuelas medias de la provincia, desarrollando una estructura de trabajo en un tronco común, por épocas, y especialidades, en el Ciclo Superior.

(5) Ésta es una institución pública, de gestión privada, religiosa (salesiana), de la capital provincial. Tiene cuatro orientaciones: Ciencias Naturales, Ciencias Sociales, Economía y Medios de Comunicación; en esta última se realizaron las entrevistas. Atiende a una población que podría pensarse como "en transición" ya que si bien concurren hijos/as (600 estudiantes, aproximadamente) de sectores medios, medios-altos (al igual que en el caso anterior, hijos/as de profesionales y funcionarios/as del gobierno de la capital 
neuquina), la gestión del establecimiento está procurando la inclusión de adolescentes del barrio -clase media, media-aja - donde está ubicada la escuela.

(6) J es una escuela inmersa en una comunidad del interior neuquino con fuertes vinculaciones con el ejército y la iglesia, atiende a una población de clase media, media baja de 500 estudiantes. Esta institución tiene orientación Técnica, con las modalidades de Electromecánica y Ciencias de la Alimentación; en esta última se efectuó el trabajo de campo.

\section{Referencias bibliograficas}

- Bottarini, R. (2007). La educación ciudadana en el vendaval político argentino. En G. Schujman e I. Siede (comps.). Ciudadanía para armar, aportes para la formación ética y política (pp. 179-226). Buenos Aires: Aique Educación.

- Cornwall, A. (2002). Making spaces, changing places: situating participation in development. Working paper 170. England: Institute of Development Studies. Consultado el 15 de septiembre de 2009, a partir de http//www.powercube.net/wpcontent/

- Cullen, C. (1997). Crítica de las razones de educar. Buenos Aires: Paidós.

- Dussel, I. y Southwell, M. (2006). De quién y para quiénes es la escuela. El Monitor de la Educación, 5, (9), 26-31.

- $\quad$ Frigerio, G. y Dicker, G. (2004). La transmisión en las sociedades, las instituciones y los sujetos. Un concepto de la educación en acción. Buenos Aires: Noveduc.

- $\quad$ Galeano, E. (1998). El libro de los abrazos. Buenos Aires: Catálogos.

- Hernández, A. (1997). Pedagogy, democracy and feminism. Rethinking the public sphere. New York: State University Press.

- Kriger, M. (2010). Jóvenes de escarapelas tomar. Escolaridad, comprensión histórica y formación política en la Argentina contemporánea. La Plata: Observatorio de Jóvenes, Comunicación y Medios y Facultad de Periodismo y Comunicación Social, Universidad Nacional de La Plata.

- Mayer, L. (2009). Hijos de la democracia. ¿Cómo piensan y viven los jóvenes? Buenos Aires: Paidós.

- Oraisón, M.M. y Pérez, A.M. (2006). Escuela y participación: el difícil camino de la construcción de ciudadanía. Revista Iberoamericana de Educación, (42), 15-29. Consultado el 15 de junio de 2007, www.rieoei.org

- Rodríguez, M. (1997). La escuela: primer espacio de actuación pública del niño. Educación y Ciudad, (3), 8-19.

- Rossi, F. (2009). La participación de las juventudes hoy. La condición juvenil y la redefinición del involucramiento político y social. Buenos Aires: Prometeo Libros.

- Viñao Frago, A. (2002). Sistemas educativos, culturas escolares y reformas. Continuidades y cambios. Madrid: Morata. 